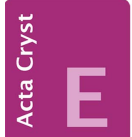
COMMUNICATIONS

ISSN 2056-9890

\section{Crystal structure of 5,5'-dibromo-3,3'-di- tert-butyl-6,6'-dimethylbiphenyl-2,2'-diol}

\author{
Rika Obata, ${ }^{a}$ Shigeru Ohba, ${ }^{a} *$ Yasuaki Einaga ${ }^{b}$ and Shigeru \\ Nishiyama ${ }^{b}$
}

${ }^{\mathrm{a}}$ Research and Education Center for Natural Sciences, Keio University, Hiyoshi 4-1-1, Kohoku-ku, Yokohama 223-8521, Japan, and ${ }^{\mathbf{b}}$ Department of Chemistry, Faculty of Science and Technology, Keio University; and JST-CREST/ACELL, Hiyoshi 3-14-1, Kohoku-ku, Yokohama 223-8522, Japan. *Correspondence e-mail: ohba@a3.keio.jp

Received 25 March 2015; accepted 28 March 2015

Edited by H. Stoeckli-Evans, University of Neuchâtel, Switzerland

The whole molecule of the title compound, $\mathrm{C}_{22} \mathrm{H}_{28} \mathrm{Br}_{2} \mathrm{O}_{2}$, is generated by twofold rotation symmetry. The dihedral angle of the biphenyl moiety is $85.05(11)^{\circ}$. The hydroxy groups show intramolecular $\mathrm{O}-\mathrm{H} \cdots \pi$ interactions without any other hydrogen-bond acceptors. In the crystal, there are no other significant intermolecular interactions present.

Keywords: crystal structure; biphenyl; axial chirality; $\mathrm{O}-\mathrm{H} \cdots \pi$ interactions.

CCDC reference: 1056738

\section{Related literature}

For the synthesis of the title compound using a transitionmetal catalyst, see: Kubota et al. (2012). For the determination of the absolute configuration of the corresponding (+)-chloro derivative, viz. S, see: Gutierrez et al. (2010). For the crystal structure of a similar compound, i.e. 5,5'-dimethoxy-6,6'-dimethylbiphenyl-2,2'-diol dichloromethane solvate, see: Guo et al. (2011).

\section{Experimental}

2.1. Crystal data

$\mathrm{C}_{22} \mathrm{H}_{28} \mathrm{Br}_{2} \mathrm{O}_{2}$

$M_{r}=484.24$

Orthorhombic, $\mathrm{Pba2}$

$a=7.3680(5) \AA$

$b=22.4243(14) \AA$

$c=6.6148(4) \AA$

\subsection{Data collection}

Bruker D8 VENTURE

diffractometer

Absorption correction: multi-scan

(SADABS; Bruker, 2014)

$T_{\min }=0.630, T_{\max }=0.773$

\subsection{Refinement}

$R\left[F^{2}>2 \sigma\left(F^{2}\right)\right]=0.031$

$w R\left(F^{2}\right)=0.065$

$S=1.06$

1966 reflections

123 parameters

1 restraint

$\mathrm{H}$-atom parameters constrained

$\Delta \rho_{\max }=0.48 \mathrm{e}^{-3}$

$=1092.91(12) \AA^{3}$

$Z=2$

Mo $K \alpha$ radiation

$\mu=3.72 \mathrm{~mm}^{-1}$

$T=299 \mathrm{~K}$

$0.16 \times 0.15 \times 0.10 \mathrm{~mm}$

9406 measured reflections 1966 independent reflections 1615 reflections with $I>2 \sigma(I)$

$R_{\text {int }}=0.036$
$\Delta \rho_{\min }=-0.21$ e $\AA^{-3}$

Absolute structure: Flack $x$ determined using 621 quotients $\left[\left(I^{+}\right)-\left(I^{-}\right)\right] /\left[\left(I^{+}\right)+\left(I^{-}\right)\right]$(Parsons et al., 2013)

Absolute structure parameter: $0.034(9)$

Table 1

Hydrogen-bond geometry $\left(\AA{ }^{\circ}\right)$.

$\mathrm{Cg}$ is the centroid of benzene ring $\mathrm{C} 3-\mathrm{C} 8$.

\begin{tabular}{lllll}
\hline$D-\mathrm{H} \cdots A$ & $D-\mathrm{H}$ & $\mathrm{H} \cdots A$ & $D \cdots A$ & $D-\mathrm{H} \cdots A$ \\
\hline $\mathrm{O} 2-\mathrm{H} 2 \cdots C g^{\mathrm{i}}$ & 0.82 & 2.54 & $3.047(5)$ & 122 \\
\hline
\end{tabular}

Symmetry code: (i) $-x+2,-y+2, z$.

Data collection: APEX2 (Bruker, 2014); cell refinement: SAINT (Bruker, 2014); data reduction: $S A I N T$; $\operatorname{program}(\mathrm{s})$ used to solve structure: SHELXS97 (Sheldrick, 2008); program(s) used to refine structure: SHELXL2014 (Sheldrick, 2015); molecular graphics: SHELXTL (Sheldrick, 2008); software used to prepare material for publication: SHELXL2014 and publCIF (Westrip, 2010).

\title{
Acknowledgements
}

We are grateful to Nippon Soda Co. Ltd for the kind gift of 4-bromo-2-tert-butyl-5-methylphenol. This research was supported by grants from the Research and Education Center for Natural Sciences, Keio University (to SO), and Keio Gijuku Academic Development Funds (to RO).

Supporting information for this paper is available from the IUCr electronic archives (Reference: SU5104). 


\section{data reports}

\section{References}

Bruker (2014). APEX2, SAINT and SADABS. Bruker AXS Inc., Madison, Wisconsin, USA.

Guo, F., Konkol, L. C. \& Thomson, R. J. (2011). J. Am. Chem. Soc. 133, 18-20. Gutierrez, E. G., Moorhead, E. J., Smith, E. H., Lin, V., Ackerman, L. K. G., Knezevic, C. E., Sun, V., Grant, S. \& Wenzel, A. G. (2010). Eur. J. Org. Chem. pp. 3027-3031.
Kubota, Y., Shirakawa, S., Inoue, T. \& Maruoka, K. (2012). Tetrahedron Lett. 53, 3739-3741.

Parsons, S., Flack, H. D. \& Wagner, T. (2013). Acta Cryst. B69, 249-259.

Sheldrick, G. M. (2008). Acta Cryst. A64, 112-122.

Sheldrick, G. M. (2015). Acta Cryst. C71, 3-8.

Westrip, S. P. (2010). J. Appl. Cryst. 43, 920-925. 


\section{supporting information}

Acta Cryst. (2015). E71, o278-o279 [https://doi.org/10.1107/S2056989015006313]

\section{Crystal structure of 5,5'-dibromo-3,3'-di-tert-butyl-6,6'-dimethylbiphenyl-2,2'- diol}

\section{Rika Obata, Shigeru Ohba, Yasuaki Einaga and Shigeru Nishiyama}

\section{S1. Synthesis and crystallization}

The synthesis of the title compound, (I), is described in Fig. 2. It was prepared using iodine-mediated coupling method from 4-bromo-2-tert-butyl-5-methylphenol. To the solution of 4-bromo-2-tert-butyl-5-methylphenol $(0.242 \mathrm{~g}, 1 \mathrm{mmol})$ in dichloromethane $\left(1 \mathrm{~mL}\right.$ ) was added $N$-iodosuccinimide (abbreviated to NIS, $0.225 \mathrm{~g}, 1 \mathrm{mmol}$ ) and $3 \% \mathrm{H}_{2} \mathrm{O}_{2}(1 \mathrm{~mL})$. After shaking $(200 \mathrm{rpm})$ the reaction mixture for $24 \mathrm{~h}$ at room temperature, it was poured into saturated $\mathrm{Na}_{2} \mathrm{~S}_{2} \mathrm{O}_{3}$ solution, and extracted with chloroform. The organic layer was washed with saturated $\mathrm{NaCl}$ and dried over anhydrous $\mathrm{Na}_{2} \mathrm{SO}_{4}$. The mixture was evaporated and purified by silica-gel column chromatography to give title compound (I) as white solid (yield: 0.138 g, 57\%). ${ }^{1} \mathrm{H}-\mathrm{NMR}\left(400 \mathrm{MHz}, \mathrm{CDCl}_{3}\right) 1.32(18 \mathrm{H}, \mathrm{s}), 1.92(6 \mathrm{H}, \mathrm{s}), 4.80(2 \mathrm{H}, \mathrm{s}), 7.47$ (2H, s). Tof-MS ES(-) Anal. 481.0357, Calcd. 481.0378 for $\mathrm{C}_{22} \mathrm{H}_{27} \mathrm{O}_{2} \mathrm{Br}_{2}$. The crystals were grown by slow evaporation from a toluene/ $n$-hexane (1/4) solution.

\section{S2. Refinement}

Crystal data, data collection and structure refinement details are summarized in the experimental table. The hydroxyl $\mathrm{H}$ atom was located from a difference Fourier map but was refined as riding (AFIX 147) with $U_{\text {iso }}(\mathrm{H})=1.5 U_{\text {eq }}(\mathrm{O})$. C-Bound $\mathrm{H}$ atoms were included in calculated positions and refined as riding: $\mathrm{C}-\mathrm{H}=0.93-0.96 \AA$ with $U_{\text {iso }}(\mathrm{H})=1.5 U_{\mathrm{eq}}(\mathrm{C})$ for methyl $\mathrm{H}$ atoms and $1.2 U_{\text {eq }}(\mathrm{C})$ for other $\mathrm{H}$ atoms. 


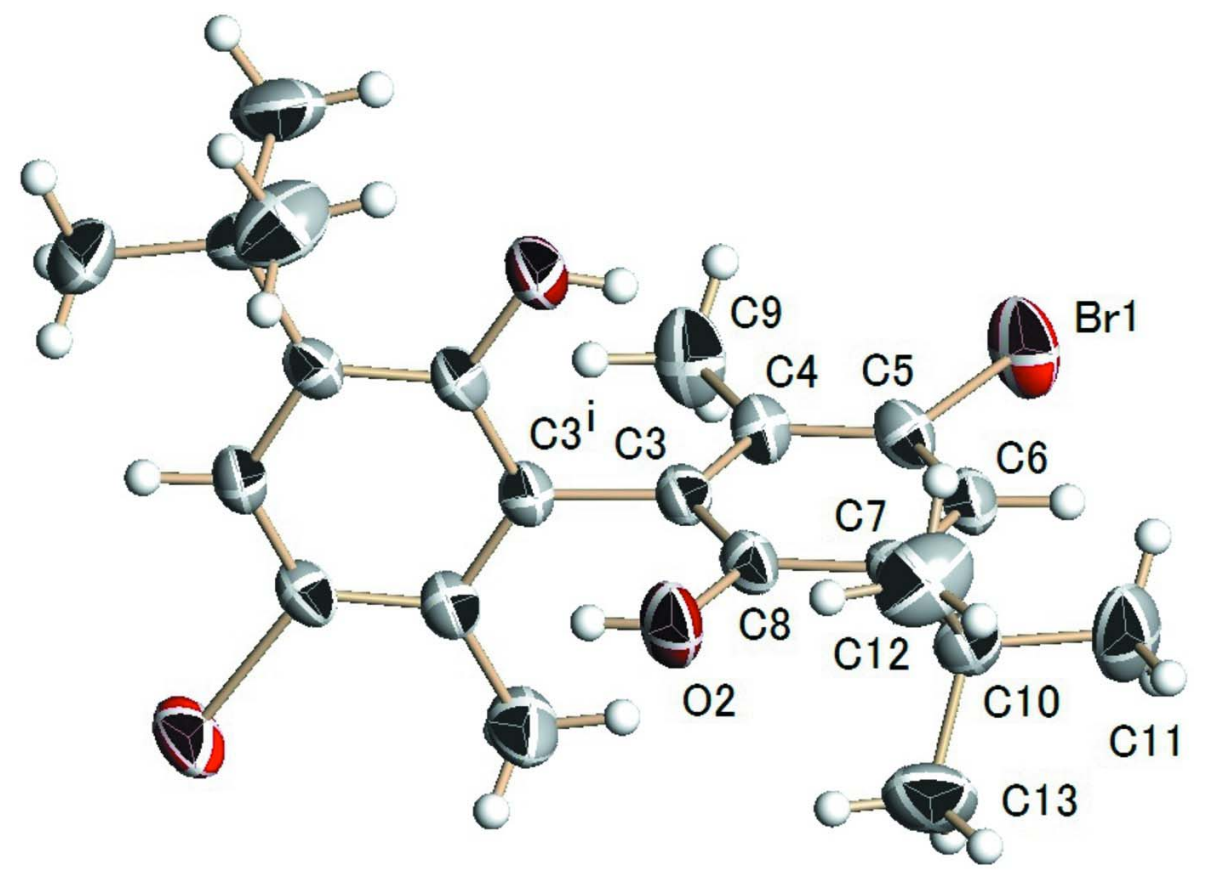

Figure 1

The molecular structure of the title compound, with atom labelling. Displacement ellipsoids are drawn at the 50\% probability level.<smiles>Cc1cc(O)c(C(C)(C)C)cc1Br</smiles>

Figure 2

The synthesis of the title compound, (I).

5,5'-Dibromo-3,3'-di-tert-butyl-6,6'-dimethylbiphenyl-2,2'-diol

Crystal data

$\mathrm{C}_{22} \mathrm{H}_{28} \mathrm{Br}_{2} \mathrm{O}_{2}$

$M_{r}=484.24$

Orthorhombic, Pba2

$a=7.3680(5) \AA$

$b=22.4243$ (14) $\AA$

$c=6.6148(4) \AA$

$V=1092.91(12) \AA^{3}$

$Z=2$

$F(000)=492$
NIS (1 eq.)

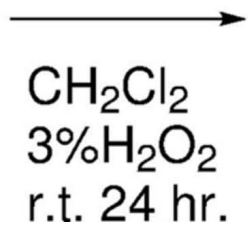

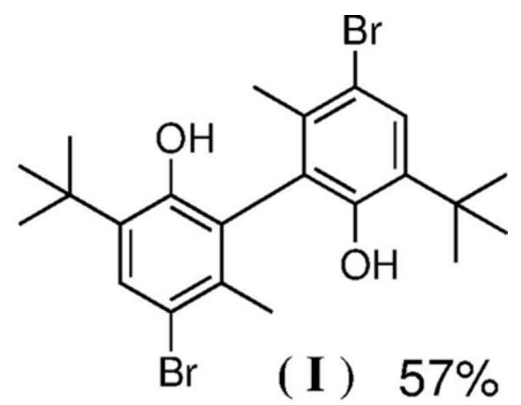




\section{Data collection}

Bruker D8 VENTURE diffractometer

Radiation source: fine-focus sealed tube $\omega$ scans

Absorption correction: multi-scan (SADABS; Bruker, 2014)

$T_{\min }=0.630, T_{\max }=0.773$

9406 measured reflections

\section{Refinement}

Refinement on $F^{2}$

Least-squares matrix: full

$R\left[F^{2}>2 \sigma\left(F^{2}\right)\right]=0.031$

$w R\left(F^{2}\right)=0.065$

$S=1.06$

1966 reflections

123 parameters

1 restraint

Primary atom site location: structure-invariant direct methods
1966 independent reflections

1615 reflections with $I>2 \sigma(I)$

$R_{\text {int }}=0.036$

$\theta_{\max }=25.3^{\circ}, \theta_{\min }=2.9^{\circ}$

$h=-8 \rightarrow 8$

$k=-26 \rightarrow 26$

$l=-7 \rightarrow 7$
Hydrogen site location: inferred from neighbouring sites

$\mathrm{H}$-atom parameters constrained

$w=1 /\left[\sigma^{2}\left(F_{\mathrm{o}}{ }^{2}\right)+(0.0176 P)^{2}+0.3778 P\right]$

where $P=\left(F_{\mathrm{o}}^{2}+2 F_{\mathrm{c}}^{2}\right) / 3$

$(\Delta / \sigma)_{\max }=0.001$

$\Delta \rho_{\max }=0.48$ e $\AA^{-3}$

$\Delta \rho_{\min }=-0.21$ e $\AA^{-3}$

Absolute structure: Flack $x$ determined using 621 quotients $\left[\left(I^{+}\right)-(I)\right] /\left[\left(I^{+}\right)+(I)\right]$ (Parsons et al., 2013)

Absolute structure parameter: 0.034 (9)

\section{Special details}

Geometry. All e.s.d.'s (except the e.s.d. in the dihedral angle between two 1.s. planes) are estimated using the full covariance matrix. The cell e.s.d.'s are taken into account individually in the estimation of e.s.d.'s in distances, angles and torsion angles; correlations between e.s.d.'s in cell parameters are only used when they are defined by crystal symmetry. An approximate (isotropic) treatment of cell e.s.d.'s is used for estimating e.s.d.'s involving 1.s. planes.

Fractional atomic coordinates and isotropic or equivalent isotropic displacement parameters $\left(\hat{A}^{2}\right)$

\begin{tabular}{lllll}
\hline & $x$ & $y$ & $z$ & $U_{\text {iso }} * / U_{\text {eq }}$ \\
\hline Br1 & $1.18668(8)$ & $0.81211(2)$ & $0.48288(15)$ & $0.0681(2)$ \\
O2 & $0.7528(4)$ & $0.98204(13)$ & $0.9897(9)$ & $0.0576(8)$ \\
H2 & 0.7777 & 1.0166 & 0.9607 & $0.086^{*}$ \\
C3 & $0.9856(6)$ & $0.96676(17)$ & $0.7429(7)$ & $0.0352(10)$ \\
C4 & $1.0903(6)$ & $0.9290(2)$ & $0.6223(7)$ & $0.0388(11)$ \\
C5 & $1.0536(6)$ & $0.8689(2)$ & $0.6397(7)$ & $0.0399(11)$ \\
C6 & $0.9212(6)$ & $0.84644(19)$ & $0.7649(7)$ & $0.0394(11)$ \\
H6 & 0.9023 & 0.8054 & 0.7685 & $0.047^{*}$ \\
C7 & $0.8150(6)$ & $0.88292(19)$ & $0.8859(7)$ & $0.0360(10)$ \\
C8 & $0.8518(6)$ & $0.94415(19)$ & $0.8707(7)$ & $0.0370(11)$ \\
C9 & $1.2322(7)$ & $0.9538(2)$ & $0.4852(15)$ & $0.0650(14)$ \\
H9A & 1.1993 & 0.9460 & 0.3473 & $0.098^{*}$ \\
H9B & 1.3467 & 0.9353 & 0.5143 & $0.098^{*}$ \\
H9C & 1.2417 & 0.9960 & 0.5058 & $0.098^{*}$ \\
C10 & $0.6668(5)$ & $0.8582(2)$ & $1.0234(8)$ & $0.0428(15)$ \\
C11 & $0.6564(7)$ & $0.7905(2)$ & $1.0111(14)$ & $0.074(2)$ \\
H11A & 0.6243 & 0.7789 & 0.8761 & $0.111^{*}$ \\
H11B & 0.5661 & 0.7762 & 1.1039 & $0.111^{*}$ \\
H11C & 0.7722 & 0.7737 & 1.0456 & $0.111^{*}$
\end{tabular}




$\begin{array}{lllll}\mathrm{C} 12 & 0.7053(8) & 0.8746(3) & 1.2449(9) & 0.0756(18) \\ \mathrm{H} 12 \mathrm{~A} & 0.8260 & 0.8623 & 1.2802 & 0.113^{*} \\ \mathrm{H} 12 \mathrm{~B} & 0.6195 & 0.8548 & 1.3311 & 0.113^{*} \\ \mathrm{H} 12 \mathrm{C} & 0.6944 & 0.9170 & 1.2619 & 0.113^{*} \\ \mathrm{C} 13 & 0.4827(6) & 0.8829(2) & 0.9625(15) & 0.0760(17) \\ \mathrm{H} 13 \mathrm{~A} & 0.4791 & 0.9249 & 0.9894 & 0.114^{*} \\ \mathrm{H} 13 \mathrm{~B} & 0.3893 & 0.8632 & 1.0387 & 0.114^{*} \\ \mathrm{H} 13 \mathrm{C} & 0.4634 & 0.8760 & 0.8208 & 0.114^{*}\end{array}$

Atomic displacement parameters $\left(\AA^{2}\right)$

\begin{tabular}{lllllll}
\hline & $U^{11}$ & $U^{22}$ & $U^{33}$ & $U^{12}$ & $U^{13}$ & $U^{23}$ \\
\hline Br1 & $0.0866(4)$ & $0.0458(3)$ & $0.0718(4)$ & $0.0118(2)$ & $0.0311(4)$ & $-0.0118(4)$ \\
O2 & $0.0646(19)$ & $0.0366(16)$ & $0.072(2)$ & $0.0049(14)$ & $0.018(3)$ & $-0.009(3)$ \\
C3 & $0.040(3)$ & $0.030(2)$ & $0.036(3)$ & $0.0016(19)$ & $-0.004(2)$ & $0.001(2)$ \\
C4 & $0.048(3)$ & $0.033(3)$ & $0.035(3)$ & $0.003(2)$ & $0.002(2)$ & $0.000(2)$ \\
C5 & $0.048(3)$ & $0.035(3)$ & $0.037(3)$ & $0.009(2)$ & $0.003(2)$ & $-0.003(2)$ \\
C6 & $0.046(3)$ & $0.028(2)$ & $0.045(3)$ & $0.002(2)$ & $-0.002(2)$ & $-0.002(2)$ \\
C7 & $0.036(3)$ & $0.034(2)$ & $0.038(2)$ & $0.008(2)$ & $-0.005(2)$ & $-0.002(2)$ \\
C8 & $0.042(3)$ & $0.031(3)$ & $0.038(2)$ & $0.006(2)$ & $0.000(2)$ & $-0.005(2)$ \\
C9 & $0.084(3)$ & $0.048(3)$ & $0.063(3)$ & $0.003(2)$ & $0.033(5)$ & $-0.003(5)$ \\
C10 & $0.035(3)$ & $0.045(3)$ & $0.048(4)$ & $-0.0010(19)$ & $0.008(3)$ & $0.003(2)$ \\
C11 & $0.070(3)$ & $0.048(3)$ & $0.104(6)$ & $-0.013(2)$ & $0.036(5)$ & $0.008(4)$ \\
C12 & $0.078(4)$ & $0.099(5)$ & $0.049(4)$ & $-0.021(4)$ & $0.010(3)$ & $0.002(3)$ \\
C13 & $0.039(3)$ & $0.086(4)$ & $0.103(5)$ & $0.005(2)$ & $0.012(4)$ & $0.020(5)$ \\
& & & & & &
\end{tabular}

Geometric parameters $\left(\AA,{ }^{\circ}\right)$

\begin{tabular}{llll}
\hline $\mathrm{Br} 1-\mathrm{C} 5$ & $1.913(4)$ & $\mathrm{C} 9-\mathrm{H} 9 \mathrm{~B}$ & 0.9600 \\
$\mathrm{O} 2-\mathrm{C} 8$ & $1.369(6)$ & $\mathrm{C} 9-\mathrm{H} 9 \mathrm{C}$ & 0.9600 \\
$\mathrm{O} 2-\mathrm{H} 2$ & 0.8200 & $\mathrm{C} 10-\mathrm{C} 13$ & $1.520(7)$ \\
$\mathrm{C} 3-\mathrm{C} 8$ & $1.394(6)$ & $\mathrm{C} 10-\mathrm{C} 11$ & $1.521(7)$ \\
$\mathrm{C} 3-\mathrm{C} 4$ & $1.396(6)$ & $\mathrm{C} 10-\mathrm{C} 12$ & $1.537(8)$ \\
$\mathrm{C} 3-\mathrm{C} 3$ & $\mathrm{C} 11-\mathrm{H} 11 \mathrm{~A}$ & 0.9600 \\
$\mathrm{C} 4-\mathrm{C} 5$ & $1.506(8)$ & $\mathrm{C} 11-\mathrm{H} 11 \mathrm{~B}$ & 0.9600 \\
$\mathrm{C} 4-\mathrm{C} 9$ & $1.379(6)$ & $\mathrm{C} 11-\mathrm{H} 11 \mathrm{C}$ & 0.9600 \\
$\mathrm{C} 5-\mathrm{C} 6$ & $1.491(8)$ & $\mathrm{C} 12-\mathrm{H} 12 \mathrm{~A}$ & 0.9600 \\
$\mathrm{C} 6-\mathrm{C} 7$ & $1.375(6)$ & $\mathrm{C} 12-\mathrm{H} 12 \mathrm{~B}$ & 0.9600 \\
$\mathrm{C} 6-\mathrm{H} 6$ & $1.386(6)$ & $\mathrm{C} 12-\mathrm{H} 12 \mathrm{C}$ & 0.9600 \\
$\mathrm{C} 7-\mathrm{C} 8$ & 0.9300 & $\mathrm{C} 13-\mathrm{H} 13 \mathrm{~A}$ & 0.9600 \\
$\mathrm{C} 7-\mathrm{C} 10$ & $1.403(6)$ & $\mathrm{C} 13-\mathrm{H} 13 \mathrm{C}$ & 0.9600 \\
$\mathrm{C} 9-\mathrm{H} 9 \mathrm{~A}$ & $1.526(6)$ & $\mathrm{C} 13-\mathrm{C} 10-\mathrm{C} 11$ & 0.9600 \\
$\mathrm{C} 8-\mathrm{O} 2-\mathrm{H} 2$ & 0.9600 & $\mathrm{C} 13-\mathrm{C} 10-\mathrm{C} 7$ & $107.7(4)$ \\
$\mathrm{C} 8-\mathrm{C} 3-\mathrm{C} 4$ & & $\mathrm{C} 11-\mathrm{C} 10-\mathrm{C} 7$ & $110.4(5)$ \\
$\mathrm{C} 8-\mathrm{C} 3-\mathrm{C}{ }^{\mathrm{i}}$ & 109.5 & $\mathrm{C} 13-\mathrm{C} 10-\mathrm{C} 12$ & $111.5(4)$ \\
$\mathrm{C} 4-\mathrm{C} 3-\mathrm{C} 3{ }^{\mathrm{i}}$ & $121.1(4)$ & $\mathrm{C} 11-\mathrm{C} 10-\mathrm{C} 12$ & $109.3(5)$ \\
$\mathrm{C} 5-\mathrm{C} 4-\mathrm{C} 3$ & $117.3(4)$ & & $107.4(5)$
\end{tabular}




\begin{tabular}{|c|c|c|c|}
\hline $\mathrm{C} 5-\mathrm{C} 4-\mathrm{C} 9$ & $123.5(4)$ & $\mathrm{C} 7-\mathrm{C} 10-\mathrm{C} 12$ & $110.5(4)$ \\
\hline $\mathrm{C} 3-\mathrm{C} 4-\mathrm{C} 9$ & $120.5(4)$ & $\mathrm{C} 10-\mathrm{C} 11-\mathrm{H} 11 \mathrm{~A}$ & 109.5 \\
\hline $\mathrm{C} 6-\mathrm{C} 5-\mathrm{C} 4$ & $123.2(4)$ & $\mathrm{C} 10-\mathrm{C} 11-\mathrm{H} 11 \mathrm{~B}$ & 109.5 \\
\hline $\mathrm{C} 6-\mathrm{C} 5-\mathrm{Br} 1$ & $116.5(3)$ & $\mathrm{H} 11 \mathrm{~A}-\mathrm{C} 11-\mathrm{H} 11 \mathrm{~B}$ & 109.5 \\
\hline $\mathrm{C} 4-\mathrm{C} 5-\mathrm{Br} 1$ & $120.3(3)$ & $\mathrm{C} 10-\mathrm{C} 11-\mathrm{H} 11 \mathrm{C}$ & 109.5 \\
\hline $\mathrm{C} 5-\mathrm{C} 6-\mathrm{C} 7$ & $122.2(4)$ & $\mathrm{H} 11 \mathrm{~A}-\mathrm{C} 11-\mathrm{H} 11 \mathrm{C}$ & 109.5 \\
\hline $\mathrm{C} 5-\mathrm{C} 6-\mathrm{H} 6$ & 118.9 & $\mathrm{H} 11 \mathrm{~B}-\mathrm{C} 11-\mathrm{H} 11 \mathrm{C}$ & 109.5 \\
\hline $\mathrm{C} 7-\mathrm{C} 6-\mathrm{H} 6$ & 118.9 & $\mathrm{C} 10-\mathrm{C} 12-\mathrm{H} 12 \mathrm{~A}$ & 109.5 \\
\hline $\mathrm{C} 6-\mathrm{C} 7-\mathrm{C} 8$ & $115.3(4)$ & $\mathrm{C} 10-\mathrm{C} 12-\mathrm{H} 12 \mathrm{~B}$ & 109.5 \\
\hline $\mathrm{C} 6-\mathrm{C} 7-\mathrm{C} 10$ & $122.2(4)$ & $\mathrm{H} 12 \mathrm{~A}-\mathrm{C} 12-\mathrm{H} 12 \mathrm{~B}$ & 109.5 \\
\hline $\mathrm{C} 8-\mathrm{C} 7-\mathrm{C} 10$ & $122.5(4)$ & $\mathrm{C} 10-\mathrm{C} 12-\mathrm{H} 12 \mathrm{C}$ & 109.5 \\
\hline $\mathrm{O} 2-\mathrm{C} 8-\mathrm{C} 3$ & $120.0(4)$ & $\mathrm{H} 12 \mathrm{~A}-\mathrm{C} 12-\mathrm{H} 12 \mathrm{C}$ & 109.5 \\
\hline $\mathrm{O} 2-\mathrm{C} 8-\mathrm{C} 7$ & $117.6(4)$ & $\mathrm{H} 12 \mathrm{~B}-\mathrm{C} 12-\mathrm{H} 12 \mathrm{C}$ & 109.5 \\
\hline $\mathrm{C} 3-\mathrm{C} 8-\mathrm{C} 7$ & $122.4(4)$ & $\mathrm{C} 10-\mathrm{C} 13-\mathrm{H} 13 \mathrm{~A}$ & 109.5 \\
\hline $\mathrm{C} 4-\mathrm{C} 9-\mathrm{H} 9 \mathrm{~A}$ & 109.5 & $\mathrm{C} 10-\mathrm{C} 13-\mathrm{H} 13 \mathrm{~B}$ & 109.5 \\
\hline $\mathrm{C} 4-\mathrm{C} 9-\mathrm{H} 9 \mathrm{~B}$ & 109.5 & $\mathrm{H} 13 \mathrm{~A}-\mathrm{C} 13-\mathrm{H} 13 \mathrm{~B}$ & 109.5 \\
\hline $\mathrm{H} 9 \mathrm{~A}-\mathrm{C} 9-\mathrm{H} 9 \mathrm{~B}$ & 109.5 & $\mathrm{C} 10-\mathrm{C} 13-\mathrm{H} 13 \mathrm{C}$ & 109.5 \\
\hline $\mathrm{C} 4-\mathrm{C} 9-\mathrm{H} 9 \mathrm{C}$ & 109.5 & $\mathrm{H} 13 \mathrm{~A}-\mathrm{C} 13-\mathrm{H} 13 \mathrm{C}$ & 109.5 \\
\hline $\mathrm{H} 9 \mathrm{~A}-\mathrm{C} 9-\mathrm{H} 9 \mathrm{C}$ & 109.5 & $\mathrm{H} 13 \mathrm{~B}-\mathrm{C} 13-\mathrm{H} 13 \mathrm{C}$ & 109.5 \\
\hline $\mathrm{H} 9 \mathrm{~B}-\mathrm{C} 9-\mathrm{H} 9 \mathrm{C}$ & 109.5 & & \\
\hline $\mathrm{C} 8-\mathrm{C} 3-\mathrm{C} 4-\mathrm{C} 5$ & $-0.5(6)$ & $\mathrm{C} 3-\mathrm{C} 3-\mathrm{C} 8-\mathrm{O} 2$ & $0.0(6)$ \\
\hline $\mathrm{C} 3-\mathrm{C} 3-\mathrm{C} 4-\mathrm{C} 5$ & $178.5(4)$ & $\mathrm{C} 4-\mathrm{C} 3-\mathrm{C} 8-\mathrm{C} 7$ & $0.1(7)$ \\
\hline $\mathrm{C} 8-\mathrm{C} 3-\mathrm{C} 4-\mathrm{C} 9$ & $179.6(5)$ & $\mathrm{C} 3^{\mathrm{i}}-\mathrm{C} 3-\mathrm{C} 8-\mathrm{C} 7$ & $-179.0(4)$ \\
\hline $\mathrm{C} 3-\mathrm{C} 3-\mathrm{C} 4-\mathrm{C} 9$ & $-1.4(7)$ & $\mathrm{C} 6-\mathrm{C} 7-\mathrm{C} 8-\mathrm{O} 2$ & $-178.9(4)$ \\
\hline $\mathrm{C} 3-\mathrm{C} 4-\mathrm{C} 5-\mathrm{C} 6$ & $0.9(7)$ & $\mathrm{C} 10-\mathrm{C} 7-\mathrm{C} 8-\mathrm{O} 2$ & $1.9(6)$ \\
\hline $\mathrm{C} 9-\mathrm{C} 4-\mathrm{C} 5-\mathrm{C} 6$ & $-179.3(6)$ & $\mathrm{C} 6-\mathrm{C} 7-\mathrm{C} 8-\mathrm{C} 3$ & $0.1(6)$ \\
\hline $\mathrm{C} 3-\mathrm{C} 4-\mathrm{C} 5-\mathrm{Br} 1$ & $179.9(3)$ & $\mathrm{C} 10-\mathrm{C} 7-\mathrm{C} 8-\mathrm{C} 3$ & $-179.1(4)$ \\
\hline $\mathrm{C} 9-\mathrm{C} 4-\mathrm{C} 5-\mathrm{Br} 1$ & $-0.2(7)$ & $\mathrm{C} 6-\mathrm{C} 7-\mathrm{C} 10-\mathrm{C} 13$ & $-118.6(5)$ \\
\hline $\mathrm{C} 4-\mathrm{C} 5-\mathrm{C} 6-\mathrm{C} 7$ & $-0.8(7)$ & $\mathrm{C} 8-\mathrm{C} 7-\mathrm{C} 10-\mathrm{C} 13$ & $60.5(6)$ \\
\hline $\mathrm{Br} 1-\mathrm{C} 5-\mathrm{C} 6-\mathrm{C} 7$ & $-179.8(3)$ & $\mathrm{C} 6-\mathrm{C} 7-\mathrm{C} 10-\mathrm{C} 11$ & $1.0(6)$ \\
\hline $\mathrm{C} 5-\mathrm{C} 6-\mathrm{C} 7-\mathrm{C} 8$ & $0.3(7)$ & $\mathrm{C} 8-\mathrm{C} 7-\mathrm{C} 10-\mathrm{C} 11$ & $-179.8(5)$ \\
\hline $\mathrm{C} 5-\mathrm{C} 6-\mathrm{C} 7-\mathrm{C} 10$ & $179.4(4)$ & $\mathrm{C} 6-\mathrm{C} 7-\mathrm{C} 10-\mathrm{C} 12$ & $120.4(5)$ \\
\hline $\mathrm{C} 4-\mathrm{C} 3-\mathrm{C} 8-\mathrm{O} 2$ & $179.0(4)$ & $\mathrm{C} 8-\mathrm{C} 7-\mathrm{C} 10-\mathrm{C} 12$ & $-60.5(6)$ \\
\hline
\end{tabular}

Symmetry code: (i) $-x+2,-y+2, z$.

Hydrogen-bond geometry $\left(A,{ }^{\circ}\right)$

$\mathrm{Cg}$ is the centroid of benzene ring $\mathrm{C} 3-\mathrm{C} 8$.

\begin{tabular}{lllll}
\hline$D-\mathrm{H} \cdots A$ & $D-\mathrm{H}$ & $\mathrm{H} \cdots A$ & $D \cdots A$ & $D-\mathrm{H} \cdots A$ \\
\hline $\mathrm{O} 2-\mathrm{H} 2 \cdots C g^{\mathrm{i}}$ & 0.82 & 2.54 & $3.047(5)$ & 122 \\
\hline
\end{tabular}

Symmetry code: (i) $-x+2,-y+2, z$. 